\title{
An Experimental Study of Superconvergence Phenomena in Finite Element Magnetics
}

\author{
Dennis Giannacopoulos and Steve McFee \\ Electrical Engineering Department, McGill University \\ 3480 University Street, Montreal, PQ, H3A 2A7, Canada
}

\begin{abstract}
The usefulness of superconvergence phenomena in practical finite element magnetics is investigated. Reports on the superconvergent characteristics of potential-based derivatives at the Gauss-Legendre quadrature points of first-order elements are tested, and Maxwell stress force calculations based on superconvergent integration contours are examined. Results from two experimental studies indicate that derivative superconvergence arises only arbitrarily, or asymptotically with increasing solution accuracy. Overall, the results of this work suggest that superconvergence phenomena, as commonly defined for first-order finite element applications in magnetics, are not apparent in practice.
\end{abstract}

\section{INTRODUCTION}

The finite element method (FEM) is a powerful numerical analysis technique which is used for solving a wide range of engineering electromagnetics problems [1]. Currently, finite element analysis (FEA) is frequently used in electromagnetic design. Typically, FEA software tools are used to numerically simulate and evaluate the performance of a new device design before constructing a physical prototype [2]. In general, finite element solutions are inherently approximate: fundamentally, the FEM is based on the principle of representing solutions to continuum problems with finite-dimensional approximations, computed over finitely discretized domains [3]. Therefore, the accuracy of a finite element solution depends directly on both the number of free parameters used to mathematically model the problem, and on how effectively those degrees of freedom (DOF) are distributed over the problem space [4]. Further, for a given finite element discretization, the solution error distribution may vary throughout the problem domain according to both the relative rate of solution variation and the corresponding concentration of DOF. In recent years, the development and application of superconvergence concepts for FEA error estimation and control has attracted a good deal of interest in the research community. In essence, superconvergence theory states that a finite element solution is inherently more accurate at certain points in the discretization than it is at others - and that these high accuracy locations are known a priori [5].

The computational analysis and design of magnetic devices frequently involves quantities related to derivatives of the underlying potential field solutions; therefore, superconvergence of derivative values of finite element potential solutions could have significant implications [6]-[8]. In fact, recent theoretical analyses on finite element superconvergence have focused on the development of superconvergent derivatives. Under certain assumptions, a number of these studies have determined that

Manuscript received January 31, 1997. This work was supported in part by the Natural Sciences and Engineering Research Council of Canada. quantities related to first-derivatives of finite element potential solutions should possess superconvergent properties. Further, these studies have concluded that the most accurate derivative values over a given finite element are to be found at the GaussLegendre quadrature points of that element [9]. Specifically, it has been argued that when a finite element potential solution computed over first-order triangles is interpolatory on the true solution to the continuum problem, the derivatives will be most accurate at the midpoints of the edges of the triangles [5],[9]. It can be shown, with the mean-value theorem of calculus, that this is not true in general, but may be valid under certain conditions. The purpose of this work, however, is to investigate the practical value of superconvergence phenomena in finite element magnetics using computational experiments, in order to better understand the true impact and merit of the concept.

\section{RESULTS}

Two benchmark magnetic systems were developed to study and evaluate superconvergence phenomena in finite element magnetics. The first involves a free space current distribution; and the second is focused on the magnetic interaction between current and iron. In each case, analytical derivatives of firstorder, potential-based, finite element solutions were examined for properties characteristic of superconvergence. In addition, for the second benchmark study, the practical implications and potential benefits of derivative superconvergence for Maxwell stress force calculations were also investigated.

\section{A. Free-space Current Distribution Test Problem}

This benchmark system was first studied in [4]. It involves a straight, infinitely long, circular-cylindrical, uniform, static current distribution in free space. A series of 20 optimal and 20 uniform first-order finite element discretizations were used to compute the magnetic vector potential over a radial neighbourhood spanning a 100-fold decay in magnitude. The optimal discretizations were obtained by solving a series of full-domain, constrained optimization problems, based on optimal functional value, for the placement of the element vertices.

Fig. 1 illustrates the optimal discretization test results. Curve A shows the distance between the theoretical derivative superconvergence points (element midpoints) and the actual ones, expressed as a percentage of half the element length. In cases when this quantity varies from element to element in a mesh, only the minimum value is plotted. Curve $B$ shows the error in the computed flux density at the theoretical superconvergence points. Both curves are derived from a series of 20 solutions, and are graphed as functions of FEA functional error. These 


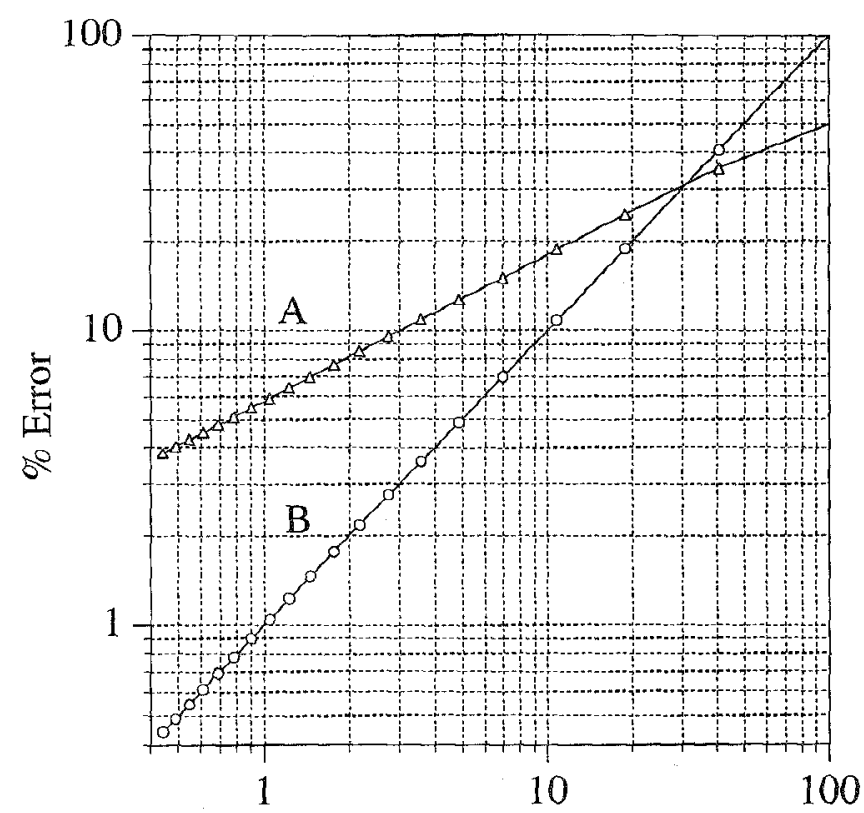

$\%$ Error in Functional Value

Fig. 1. Evaluation of derivative superconvergence properties for optimal firstorder discretizations of free-space current.

error quantities do not vary from element to element over any optimal mesh. Further, the computed potential values are exact at the element vertices for the optimal discretizations: the firstorder FEA solutions are interpolatory on the true solution to the continuum problem. Therefore, according to superconvergence theory, the Gauss-Legendre quadrature points should provide the most accurate derivatives within the elements. However, as described by Fig. 1, the computed derivatives are exact at other locations within the elements, which vary with global solution

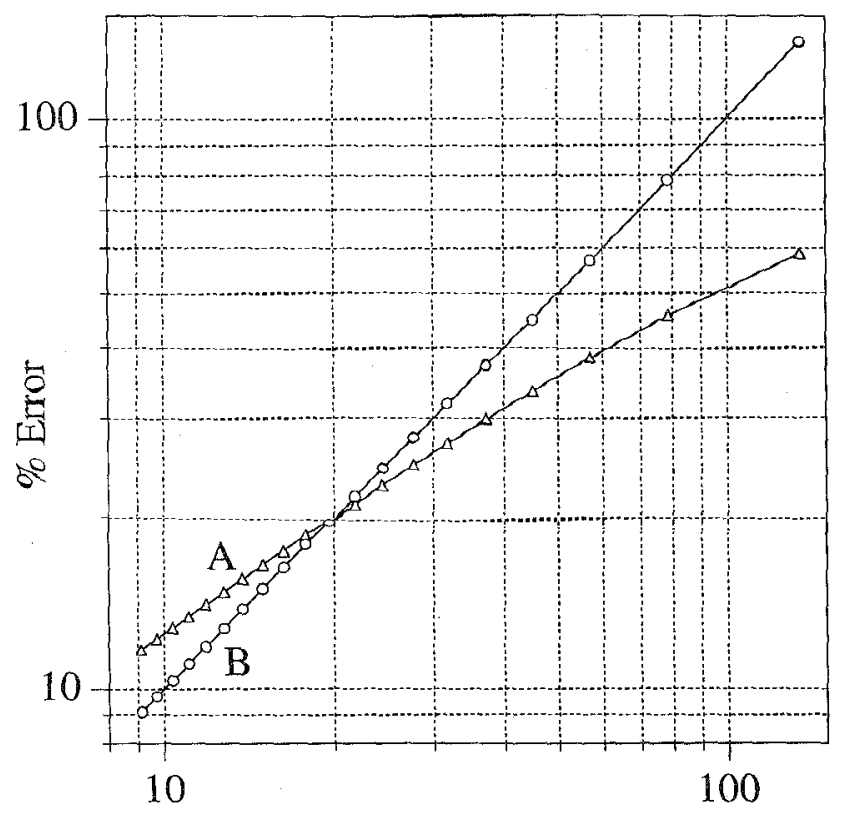

$\%$ Error in Functional Value

Fig. 2. Evaluation of derivative superconvergence properties for uniform firstorder discretizations of free-space current. accuracy. Also, by superconvergence theory, the error in the computed flux density at these Gauss-Legendre points should converge more rapidly than the global solution error. But, as seen in Fig. 1, the ratio of these two errors is unity. The results given in Fig. 2 for the uniform meshes are similar, except the error in the position of the theoretical superconvergence points (curve A) varies from element to element within each mesh.

\section{B. Magnetic Force Test Problem}

The test system investigated in this section is closely based on the magnetostatic force benchmark problem defined in [10] and revisited in [11]. The system is translationally symmetric and essentially two-dimensional. It consists of three infinitely long parallel rails fixed in free space. The system specifics are provided in Fig. 3. The infinite extent of the system in the $x-y$ plane was artificially truncated with a zero potential boundary condition at $x, y= \pm 1.2 \mathrm{~m}$ for all FEA. The first-order results were obtained using a discretization of 987 triangles and 520 DOF. The converged results were confirmed using repeated refinements of densely meshed fourth-order discretizations.

The validity and value of superconvergence phenomena for practical FEA applications were investigated and analyzed in two ways. First, the impact of superconvergence on Maxwell stress (MS) force calculation was examined. The goal was to calculate the net magnetic force acting on the iron rail, per unit length. Following standard practice [10], only MS contours in the annulus defined by Fig. 3 were used in this experiment (an example is illustrated). In this case, superconvergence theory predicts that the central contour, which intersects the midside points of every triangle in the annulus, should render the most accurate force estimate. As an aside, this result is unforeseen by the current theory of MS calculations and first-order FEA [11], which proves that the central contour MS result is simply the average of the MS forces evaluated over all such contours within the annulus. For this system, the central contour force estimate (superconvergence result) gave a $10.5 \%$ error, while a second MS calculation based on a contour defined $1 \mathrm{~mm}$ to the inside of the outer boundary of the same annulus of elements resulted in only a $3.9 \%$ error in the force estimate.

The second experiment examined the relative accuracy of solution potential derivatives at the superconvergence points. For this system, the two potential derivatives of most practical interest are $B_{x}$ and $B_{y}$. Both field component derivatives were evaluated analytically from the first-order potential solution at all points along the contour $a-b$, defined by Fig. 3 . The contour was purposefully located in a high field variation region, and defined to intersect the midside (superconvergence) points of every triangle along its path. The variation and accuracy of these derivatives over each elemental segment of the complete contour, including at all 7 superconvergence points, are given by Figs. 4 and 5 . These results suggest that, for practical firstorder FEA applications, the superconvergence points are just as likely to reveal locations of maximum derivative error, as they are to highlight points of superior derivative accuracy!

Both the MS force and $\boldsymbol{B}$-field derivative experiments failed to provide any support for the existence of superconvergence phenomena in practical first-order FEA applications. 


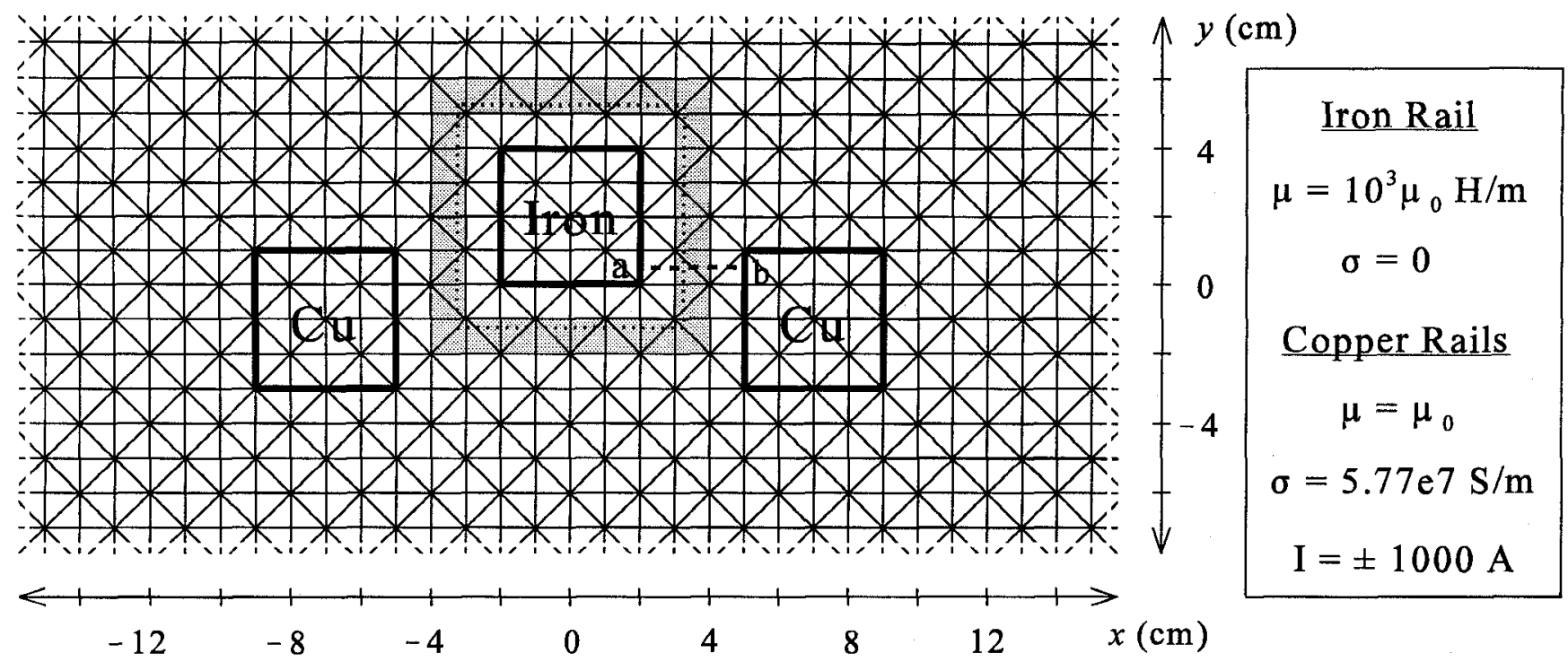

Fig. 3. Magnetic force test problem description and definition: system geometry and dimensions; material properties; excitation currents; and local finite element discretization. Shaded annulus and dotted contour therein are for MS force analysis; dashed contour from "a" to "b" is for $B x$ and $B y$ analyses.

\section{CONCLUSIONS}

The results of this experimental study indicate that superconvergence phenomena, as commonly defined for first-order FEA applications, are not apparent in practice. In particular, these investigations have demonstrated that, for the first-order FEA cases considered, derivatives of potential-based solutions are not generally more accurate at the Gauss-Legendre points. While these points yielded the lowest derivative error in some elements, they also rendered the worst derivative estimates in numerous others. However, it was observed that, under certain conditions, the properties attributed to superconvergence for FEA applications appear to become increasingly evident with increasing solution accuracy. This secondary result suggests that further theoretical and experimental studies may be useful to clearly establish the practical value of superconvergence in engineering FEA applications.

\section{REFERENCES}

[1] J. Jin, The Finite Element Method in Electromagnetics, Wiley, New York, 1993.

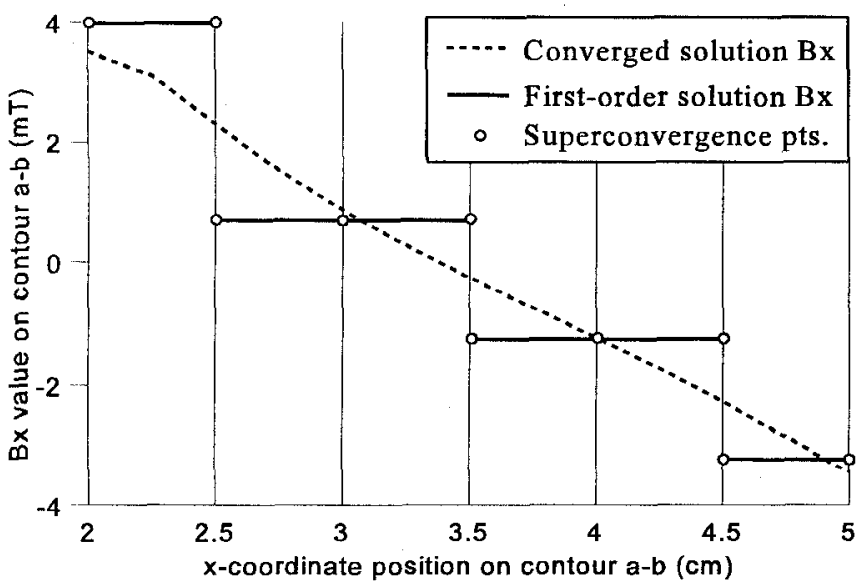

Fig. 4. Variation of $x$-component of magnetic flux density $B$ on contour $a-b$ defined in Fig. 3. Bx obtained as derivative of first-order potential solution.
[2] J.C. Sabonnadiere and A. Konrad, "Computing EM fields", IEEE Spectrum, Vol. 29(11), pp. 52-56, 1992.

[3] O.C. Zienkiewicz and R.L. Taylor, The Finite Element Method, $4^{\text {th }}$ Edition, McGraw-Hill, London, 1989.

[4] D. Giannacopoulos and S. McFee, "Towards optimal $h-p$ adaption near singularities in finite element electromagnetics", IEEE Trans. Magn., Vol. 30(5), pp. 3523-3526, 1994.

[5] M. Krizek, "Superconvergence phenomena in the finite element method", Comp. Meth. Appl. Mech. Eng., Vol. 16, pp. 157-163, 1994.

[6] P.P. Silvester and D. Omeragic, "A two-dimensional Zhu-Zienkiewicz method for gradient recovery from finite-element solutions", COMPEL, Vol. 12(3), pp. 191-204, 1993.

[7] S.X. Chen et al., "Super convergence theory and its application to precision force calculation", IEEE Trans. Magn., Vol. 32(5), pp. 42754277, 1996.

[8] P.P. Silvester and D. Omeragic, "A comparative experimental study of differentiation methods on finite elements", Int. J. Appl. Eletromagn. Mat., Vol. 4, pp. 123-136, 1993.

[9] R.J. Mackinnon and G.F. Carey, "Superconvergent derivatives: A Taylor series analysis", Int. J. Num. Meth. Eng., Vol, 28, pp. 489-509, 1989.

[10] S. McFee and D.A. Lowther, "Towards accurate and consistent force calculation in finite element based computational magnetostatics", IEEE Trans. Magn., Vol. 23(5), pp. 3771-3773, 1987.

[11] S. McFee, J.P. Webb and D.A. Lowther, "A tunable volume integration formulation for force calculation in finite-element based computational magnetostatics", IEEE Trans. Magn., Vol. 24(1), pp. 439-442, 1988.

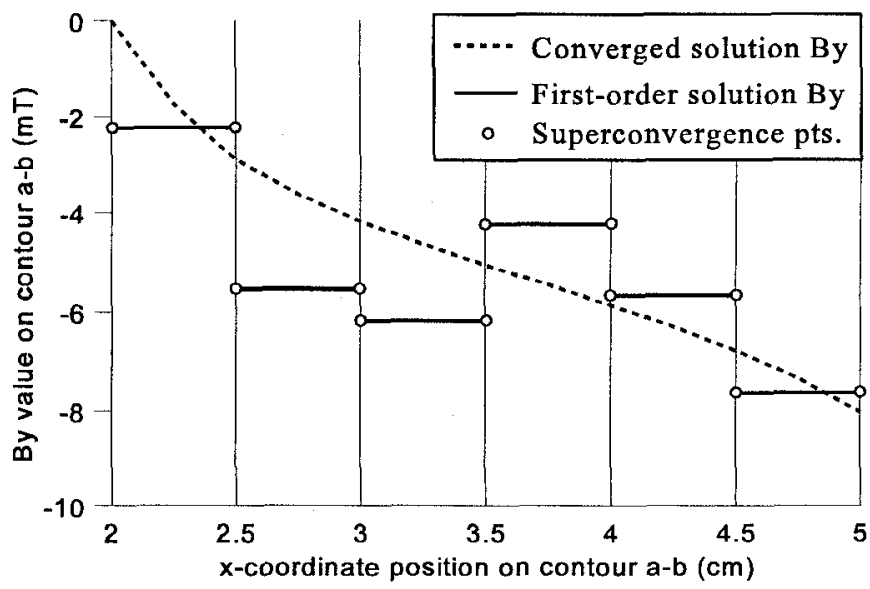

Fig. 5. Variation of $y$-component of magnetic flux density $\boldsymbol{B}$ on contour a-b defined in Fig. 3. By obtained as derivative of first-order potential solution. 